\title{
Inhibition of hedgehog signalling prevents experimental fibrosis and induces regression of established fibrosis
}

\author{
Angelika Horn, ${ }^{1}$ Trayana Kireva, ${ }^{1}$ Katrin Palumbo-Zerr, ${ }^{1}$ Clara Dees, ${ }^{1}$ Michal Tomcik, ${ }^{1,2}$ \\ Cinzia Cordazzo, ${ }^{1,3}$ Pawel Zerr, ${ }^{1}$ Alfiya Akhmetshina, ${ }^{1}$ Martial Ruat, ${ }^{4}$ Oliver Distler, ${ }^{5}$ \\ Christian Beyer, ${ }^{1}$ Georg Schett, ${ }^{1}$ Jörg H W Distler ${ }^{1}$
}

- Additional figures are published online only. To view the files, please visit the journal online (http://ard.bmj.com/ content/71/5.toc).

'Department of Internal Medicine III and Institute for Clinical Immunology, University of Erlangen-Nuremberg, Erlangen, Germany ${ }^{2}$ Institute of Rheumatology and Connective Tissue Research Laboratory, Department of Rheumatology of the First Faculty of Medicine, Charles University in Prague, Prague, Czech Republic

${ }^{3}$ Laboratory of Respiratory Cell Biology, Dipartimento Cardiotoracico e Vascolare, University of Pisa, Pisa, Italy ${ }^{4}$ CNRS, UPR3294, Laboratoire de Neurobiologie et Developpement, Institut de Neurobiologie Alfred Fessard, Gif-sur-Yvette, France

${ }^{5}$ Center of Experimental Rheumatology and Zurich Center of Integrative Human Physiology, University Hospital Zurich, Zurich, Switzerland

\section{Correspondence to}

Jörg H W Distler, Department of Internal Medicine 3 and Institute for Clinical Immunology, University of Erlangen-Nuremberg, Erlangen 91054, Germany: joerg.distler@uk-erlangen.de

Received 9 October 2011 Accepted 27 January 2012 Published Online First 8 March 2012

\section{ABSTRACT \\ Objectives Tissue fibrosis is a leading cause of death in patients with systemic sclerosis (SSc). Effective antifibrotic treatments are not available. Here, the authors investigated inhibition of hedgehog signalling by targeting Smoothened (Smo) as a novel antifibrotic approach.}

Methods The activation status of the hedgehog pathway was assessed by immunohistochemistry for Gli transcription factors and by quantification of hedgehog target genes. Hedgehog signalling was inhibited by the selective inhibitor LDE223 and by small interfering RNA against Smo in the models of bleomycin-induced dermal fibrosis and in tight-skin-1 mice.

Results Hedgehog signalling is activated in SSc and in murine models of SSc. Inhibition of Smo either by LDE223 or by small interfering RNA prevented dermal thickening, myofibroblast differentiation and accumulation of collagen upon challenge with bleomycin. Targeting Smo also exerted potent antifibrotic effects in tight-skin-1 mice and did prevent progression of fibrosis and induced regression of pre-established fibrosis.

Conclusions Inhibition of hedgehog signalling exerted potent antifibrotic effects in preclinical models of SSc in both preventive and therapeutic settings. These findings might have direct translational implications because inhibitors of Smo are already available and yielded promising results in initial clinical trials.

\section{INTRODUCTION}

The most characteristic feature of scleroderma is the massive accumulation of extracellular matrix in the skin and in affected organs. ${ }^{1}$ The progressive fibrosis impairs the function of the affected organs and results in high morbidity and mortality. However, effective antifibrotic treatments are not available for clinical use.

The hedgehog pathway is considered as a major morphogen pathway with crucial roles in embryonic development. ${ }^{2}$ In contrast to embryonic development, hedgehog signalling remains silent in most adult tissues. In the absence of hedgehog ligands, the hedgehog receptor Patched homologue 1 (Ptch-1) prevents activation of the hedgehog pathway by inhibition of the coreceptor Smoothened (Smo). However, mutations of Ptch-1 or uncontrolled expression of hedgehog proteins can result in inadequate activation of hedgehog signalling in adults. In these cases, the inhibition of Smo by Ptch-1 is released. Active Smo then induces stabilisation Gli transcription factors such as Gli-1 and Gli-2, which stimulate the transcription of hedgehog target genes such as ptch-1, ptch-2 and cyclin D1. ${ }^{3}$ Considering the important role of hedgehog signalling for cellular differentiation, it may not be surprising that aberrant activation of the hedgehog pathway has been implicated in various diseases. ${ }^{4}$ We have recently shown that the hedgehog ligand sonic hedgehog (Shh) is overexpressed in systemic sclerosis (SSc) and that the Gli transcription factors accumulate in SSc. Moreover, Shh potently induced fibroblast activation in vitro and in vivo. ${ }^{5}$

Several pharmacological strategies to inhibit hedgehog signalling have been developed over the last years. Among those, targeting of Smo seems most promising. Several inhibitors of Smo have advanced to clinical trials. Recent results have demonstrated that targeting of Smo is well tolerated in adults despite its crucial roles during embryonic development. ${ }^{7}$ After previously demonstrating that Shh stimulates fibroblast activation in SSc, we now aimed to investigate the inhibition of hedgehog signalling as a potential novel antifibrotic approach in murine models of scleroderma.

\section{METHODS}

\section{Inhibition of Smo by LDE223}

Smo was targeted with LDE223 (kindly provided by Novartis, Basel, Switzerland), a selective inhibitor of the hedgehog pathway. LDE223 was dissolved in polyethylene glycol 300 supplemented with $10 \%$ dimethyl sulphoxide and administered once daily at doses of $10 \mathrm{mg} / \mathrm{kg} / \mathrm{day}$ or $40 \mathrm{mg} /$ $\mathrm{kg} /$ day. Bleomycin-induced dermal fibrosis was induced according to established protocols. ${ }^{8} 9$ LDE223 or small interfering RNA (siRNA) was applied during the whole 3 -week period of bleomycin challenge. After 3 weeks of treatment, the fibrotic changes were quantified. In addition, tight-skin-1 (tsk-1) model was evaluated as previously described. ${ }^{10} 11$ Tsk-1 mice were treated with LDE223 from week 5 to week 10 and killed thereafter. Sham treatment was performed with $10 \%$ dimethyl sulphoxide/polyethylene glycol 300, the solvent of LDE223. 


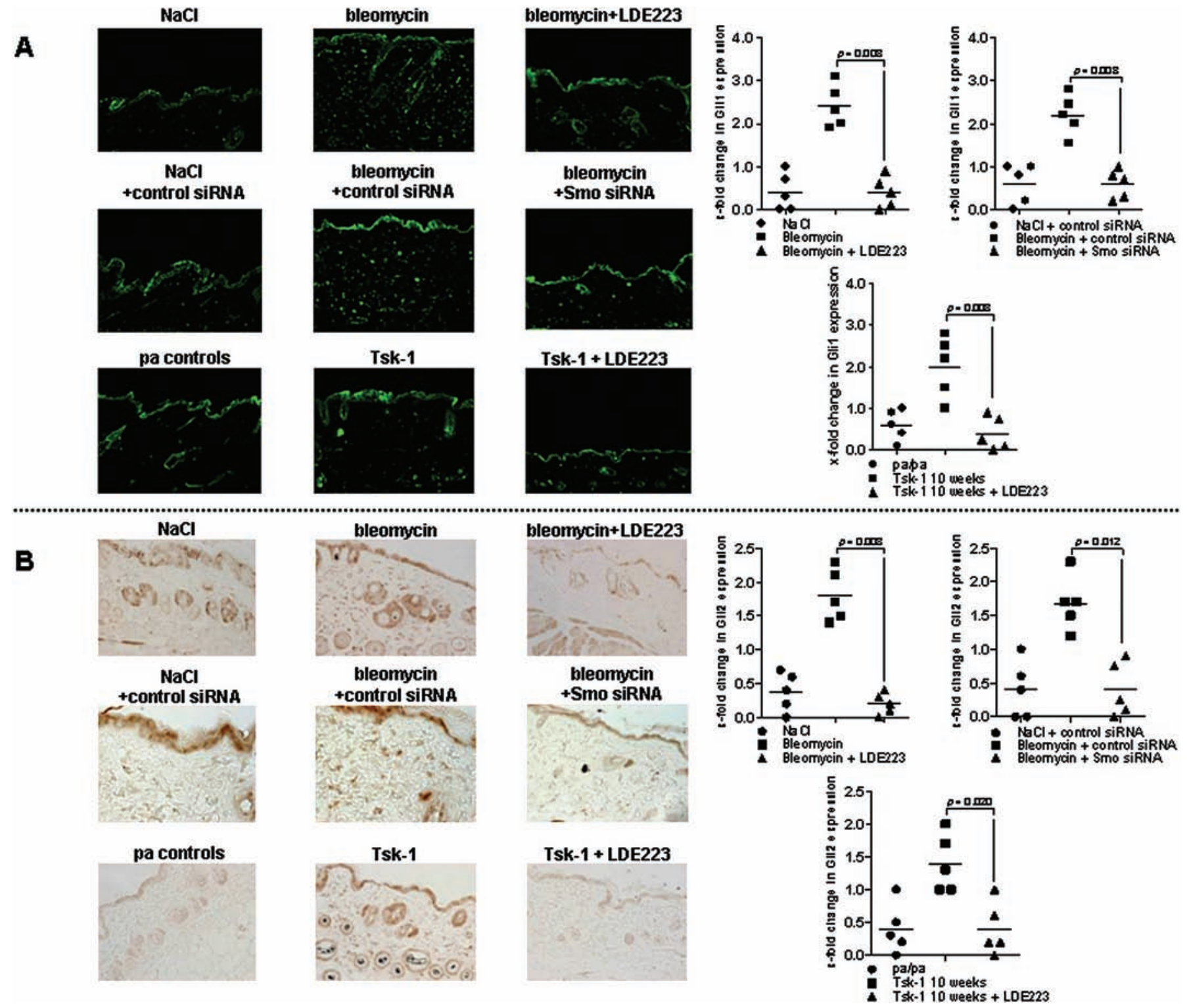

Figure 1 The aberrant activation of hedgehog signalling in experimental models of fibrosis is abrogated by LDE223 or by siRNA against Smo. Accumulation of the transcription factors Gli-1 (A) and Gli-2 (B) in the mouse model of bleomycin-induced skin fibrosis and in tsk-1 mice can be prevented by LDE223 or by siRNA-mediated knockdown of Smo. Semiquantitative analyses (with a scale of 0 for no staining to 3 for intense staining in the dermis) summarised the overexpression of Gli-1 and Gli-2 in five independent experiments per group. Representative images are shown at 400 fold magnification. siRNA, small interfering DNA; Smo, Smoothened; tsk-1, tight-skin-1.

In vivo transfection with siRNAs against Smo in bleomycin-induced skin fibrosis

Complexes of siRNA and atelocollagen (Koken, Tokyo, Japan) were prepared as previously described. ${ }^{12}$ siRNA duplexes (sense 5'-UGCCCAAGUGUGAGAAUGAUU-3', antisense 5'-PUCAUUCUCACACUUGGGCAUU-3') from Dharmacon (Lafayette, Colorado, USA) were used. Non-targeting siRNA duplexes served as controls. After confirmation of an effective knockdown of Smo in vitro, atelocollagen/siRNA complexes were injected intracutaneously once weekly into bleomycinchallenged skin areas.

\section{Histological analysis}

The dermal and hypodermal thicknesses were determined on H\&E-stained sections as previously described. ${ }^{13}$
Immunohistochemistry for Gli-1, Gli-2 and $\alpha$-smooth muscle actin

Staining for $\alpha$-smooth muscle actin as a marker for myofibroblasts was performed as previously described. ${ }^{14}$ The expression of Gli-1 and Gli-2 was detected by polyclonal rabbit-antihuman Gli-1 antibodies (GeneTex, Irvine, California, USA) and rabbit-antihuman Gli-2 antibodies (Abcam, Cambridge, UK). Respective isotype antibodies were used as negative controls. The dermal staining was semiquantitatively analysed with 0 for absent staining, 1 for low-grade dermal staining, 2 for moderate staining and 3 for intense staining.

\section{Hydroxyproline assay}

The total collagen content in lesional skin was determined using hydroxyproline assays. ${ }^{11}$ 
A

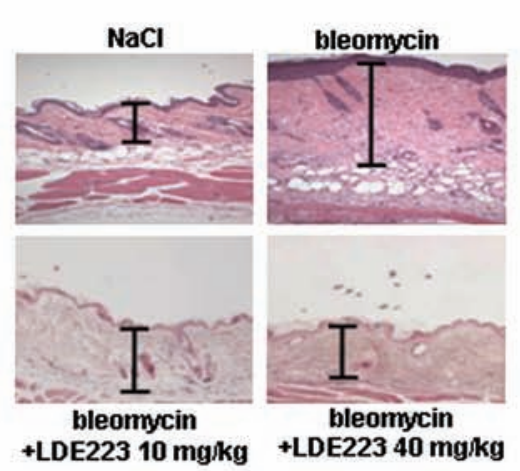

B

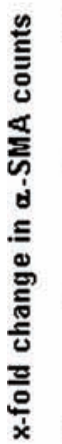

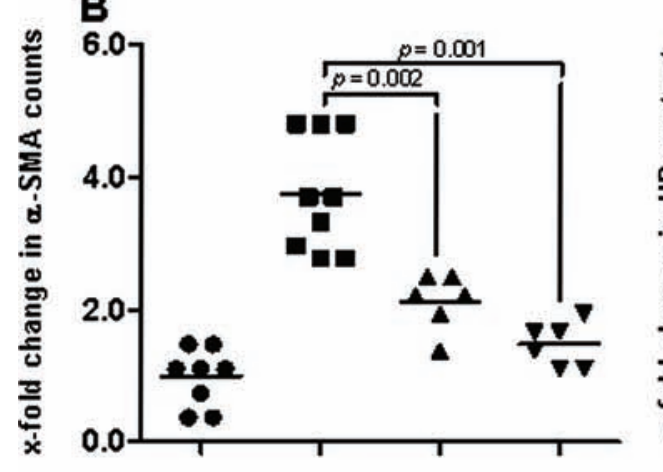

- $\mathrm{NaCl}$

- Bleomycin

- Bleomycin + LDE223 $10 \mathrm{mg} / \mathrm{kg}$

v Bleomycin + LDE223 $40 \mathrm{mg} / \mathrm{kg}$
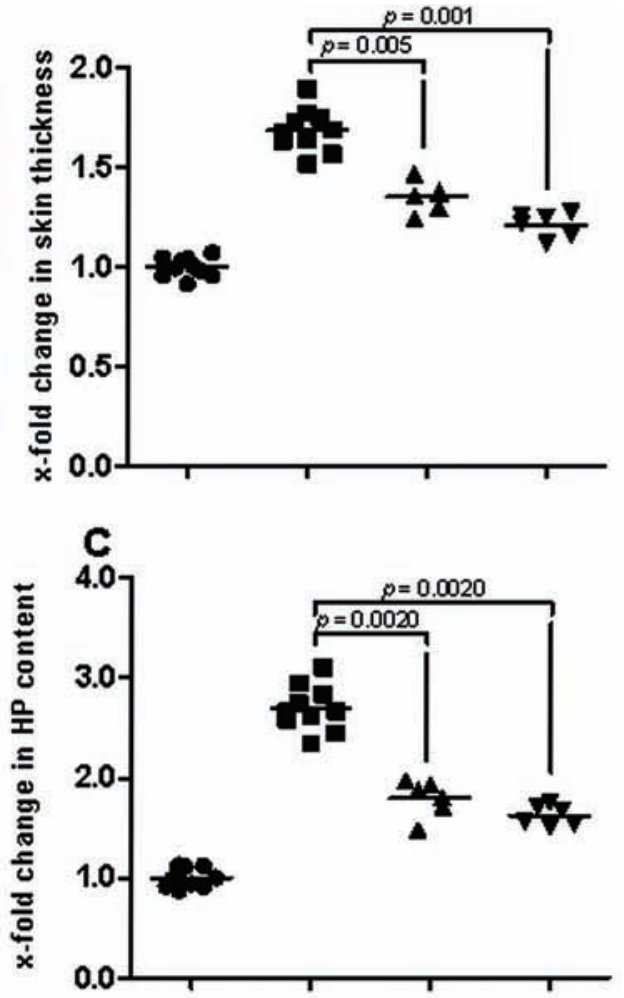
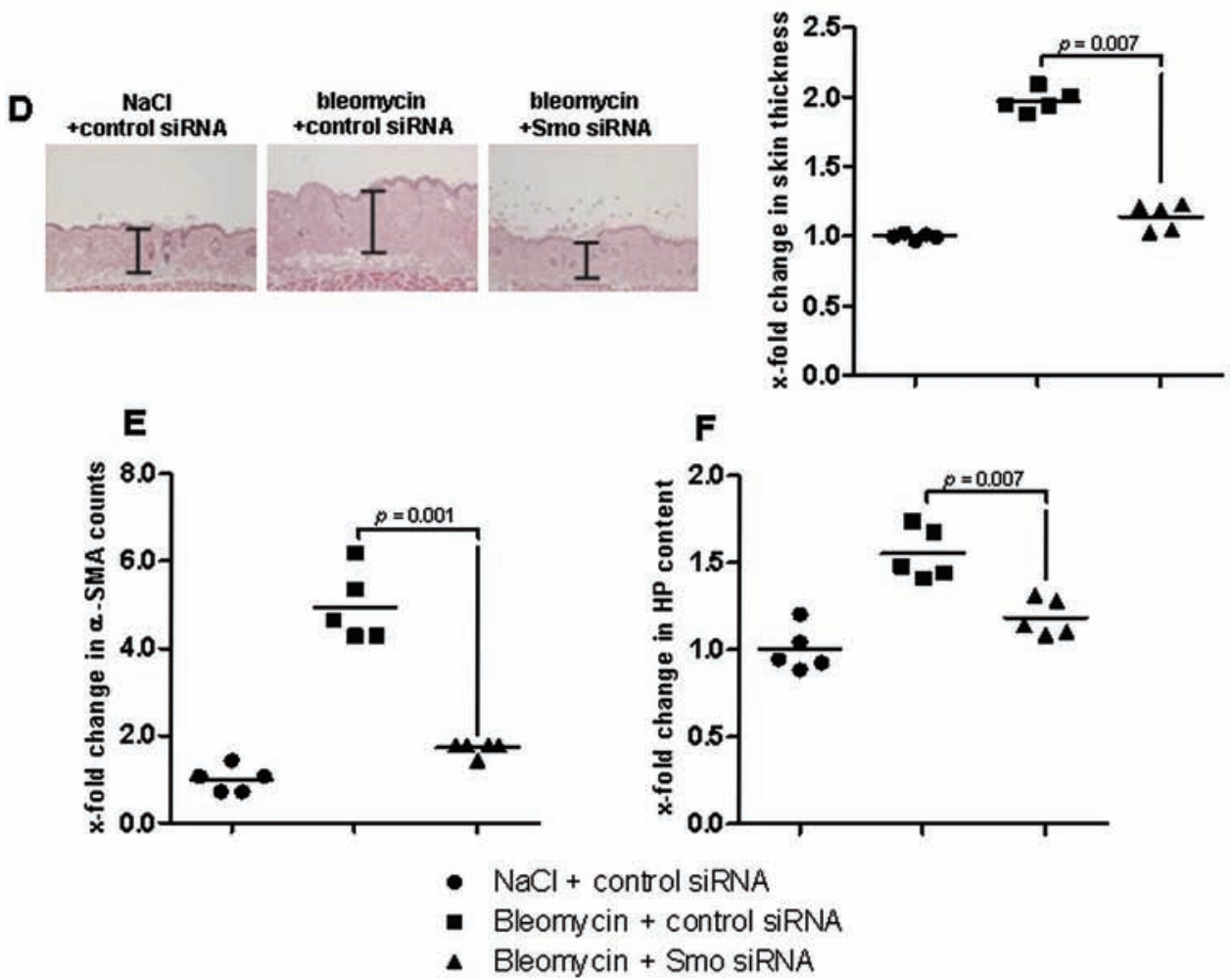

Figure 2 Inhibition of Smo prevents bleomycin-induced dermal fibrosis. Treatment with LDE223 prevents experimental fibrosis with reduction of dermal thickening (A), myofibroblast differentiation (B) and hydroxyproline content (C). Representative H\&E-stained tissue sections are shown at 100-fold magnification. $n=10$ for $\mathrm{NaCl}$ and bleomycin; $n=6$ for each group treated with LDE223. siRNA-mediated knockdown of Smo prevents dermal thickening (D) and differentiation of resting fibroblasts into myofibroblasts $(E)$ and increases hydroxyproline content (F), thereby confirming that the antifibrotic effects of LDE223 are not mediated by off-target effects. $n=5$ for each group. Representative sections are shown at 100-fold magnification. siRNA, small interfering DNA; Smo, Smoothened. 
A
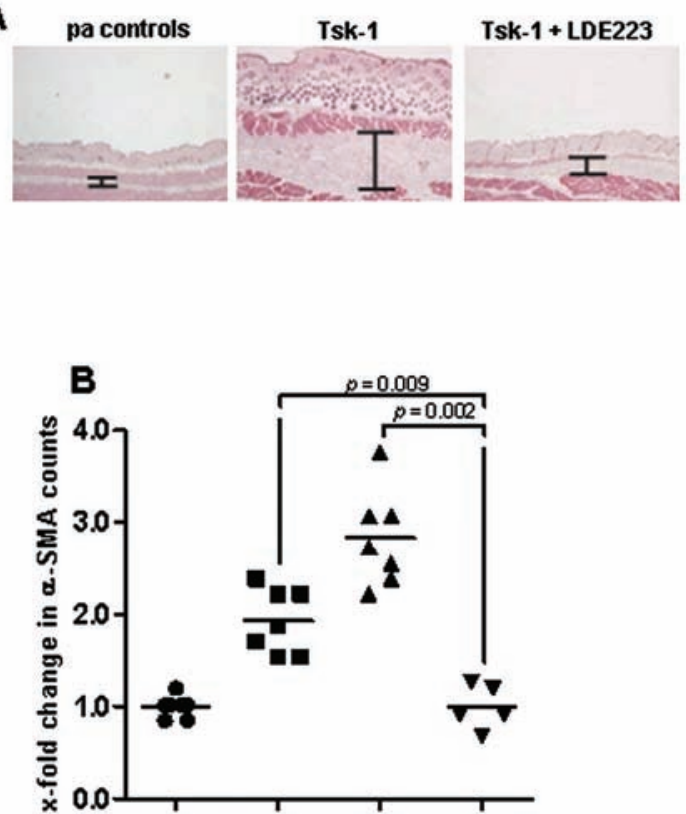
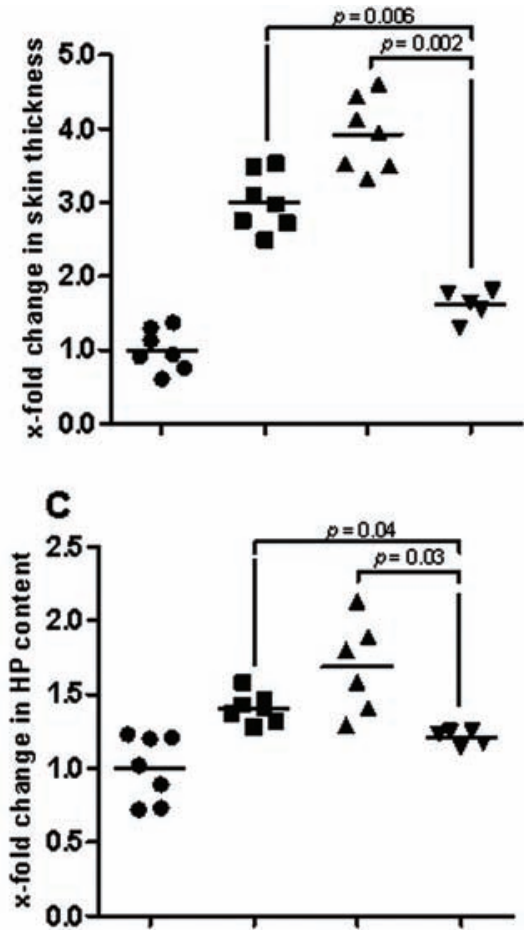

- paipa

- Tsk-1 5 weeks

A Tsk-1 10 weeks

v Tsk-1 10 weeks + LDE223

Figure 3 LDE223 induces regression of fibrosis in the tsk-1 mouse model. Treatment of tsk-1 mice with LDE223 initiated at an age of 5 weeks prevents progression of fibrosis and induces regression of fibrosis and reduces hypodermal thickness $(A)$, numbers of myofibroblasts $(B)$ and the hydroxyproline content $(C)$ to below the levels of 5-week-old tsk-1 mice. $n=7$ for all sham-treated groups; $n=5$ for the group treated with LDE223. Representative sections are shown at 40 -fold magnification. tsk-1, tight-skin-1.

\section{Quantitative real-time PCR}

Gene expression was quantified by SYBR Green real-time PCR using the ABI Prism 7300 Sequence Detection System (Applied Biosystems, Foster City, California, USA). ${ }^{15} 16$ The following primer pairs were used: murine Smo: forward primer, 5'-TGCCACCAGAAGAACAAGC-3'; reverse primer, $5^{\prime}$-GGAGATCTCTGCCTCAACCA-3'; murine $\beta$-actin: forward primer, 5'-TCT TTG ATG TCA CGC ACG AT-3'; reverse primer, 5'-TAC AGC TTC ACC ACC ACA-3'; murine ptch-1: forward primer, 5'-AGC GTG GCC CTC ACC TCC AT-3'; reverse primer, 5'-ACG CTC GCA GGG CAG GGA TA-3'; murine ptch-2: forward primer, $5^{\prime}$-CGG TGC TGA CAC TCT TGG GCC-3'; reverse primer, 5'-AGA GCA GCA CAG GCA GCA GC-3'; murine cyclin D1: forward primer, 5'-CAG AGG CGG ATG AGA ACA AG-3'; reverse primer, 5'-GTT GTG CGG TAG CAG GAG AG-3'.

\section{Statistics}

Data are expressed as dot blots with mean. The Mann-Whitney $\mathrm{U}$ test for non-related samples was used for statistical analyses. A $p$ value of less than 0.05 was considered statistically significant.

\section{RESULTS}

Inhibition of Smo abrogates the aberrant activation of hedgehog signalling in experimental models of dermal fibrosis

We first analysed whether the activation of hedgehog signalling with accumulation of the downstream transcription factor Gli-1 and Gli-2 in human scleroderma is mimicked in murine models A prominent staining for Gli-1 and Gli-2 was found in bleomycin-challenged mice by immunohistochemistry. Gli-1 (figure 1A) and Gli-2 (figure 1B) were detected in the dermis and epidermis of mice challenged with bleomycin and in tsk-1 mice. In contrast, the expression of Gli-1 and Gli-2 was restricted to the epidermal cells, hair follicles and glands but was absent in dermal cells in non-fibrotic control mice (figure 1A,B). Thus, Gli transcription factors are selectively upregulated in the dermis but not in epidermal structures. The activation of hedgehog signalling in bleomycin-induced dermal fibrosis was further confirmed by detection of increased mRNA levels of the hedgehog target genes ptch-1, ptch-2 and cyclin-D (supplementary figure 1A,B). Treatment with LDE223 prevented the increase in Gli-1 and Gli-2 (figure 1A,B) as well as the upregulation of hedgehog target genes (supplementary figure $1 \mathrm{~A}, \mathrm{~B}$ ) in both models, demonstrating that LDE223 effectively abolished the aberrant activation of the hedgehog pathway.

\section{Inhibition of Smo by LDE223 prevents bleomycin-induced dermal fibrosis}

LDE223 was well tolerated and no differences in body weight or other obvious signs of toxicity were observed. Treatment with LDE223 reduced dermal thickening in a dose-dependent manner by $71 \pm 2 \%(\mathrm{p}=0.001$ ) (figure $2 \mathrm{~A}$ ). The myofibroblast counts were reduced by $87 \pm 7 \%$ and the hydroxyproline content decreased by $68 \pm 18 \%$ in mice treated with LDE223 ( $p=0.02$ and $p=0.001$, respectively) (figure $2 \mathrm{~B}, \mathrm{C}$ ). 


\section{siRNA-mediated knockdown of Smo prevents experimental fibrosis}

To confirm that the antifibrotic effects of LDE223 are not due to off-targeting effects, we knocked down the expression of Smo locally in the skin. siRNA against Smo efficiently decreased the mRNA levels of Smo during the complete observation period with minimal decreases of $52 \pm 10 \%$ at the end of the injection period ( $p=0.04)$. siRNA-mediated knockdown of Smo reduced dermal thickening by $87 \pm 3 \%$ compared to mice injected with non-targeting siRNA ( $\mathrm{p}=0.016$ ) (figure $2 \mathrm{D})$ ). The number of myofibroblasts and the hydroxyproline content decreased by $83 \pm 4 \%$ and $66 \pm 7 \%$, respectively, in mice injected with Smo siRNA $(\mathrm{p}=0.007$ and $\mathrm{p}=0.04$ ) (figure $2 \mathrm{E}, \mathrm{F})$.

\section{Inhibition of Smo corrects the tsk-1 phenotype}

We next evaluated the effects of LDE223 in the tsk-1 mouse model. Skin fibrosis was already observed in 5-week-old tsk-1 mice and progressed over time with significantly increased fibrosis in 10-week-old mice (figures 3A-C). Treatment with LDE223 did prevent further progression of fibrosis and potently reversed the fibrotic changes. The hypodermal thickness in mice treated with LDE223 was significantly reduced by $80 \pm 5 \%$ compared to sham-treated 10-week-old tsk-1 mice $(p=0.002)$. Moreover, the hypodermal thickness in tsk-1 mice treated with LDE223 from an age of 5 weeks to an age of 10 weeks was also significantly reduced compared to 5 -week-old tsk- 1 mice $(161 \pm 5 \%$ increase vs $373 \pm 10 \%$ increase compared to mice not carrying the tsk-1 mutation, $\mathrm{p}=0.006$ ) (figure $3 \mathrm{~A}$ ). Moreover, LDE223 treatment initiated at an age of 5 weeks completely prevented myofibroblast differentiation (figure 3B) and decreased the hydroxyproline content below pretreatment levels (figure 3C).

\section{DISCUSSION}

We showed previously that hedgehog signalling is activated in SSc and induces an activated phenotype in fibroblasts. ${ }^{5}$ Here, we demonstrate that inhibition of hedgehog signalling by targeting Smo exerts potent antifibrotic effects in preclinical models. Targeting of Smo by siRNA or by the selective small-molecule inhibitor LDE223 effectively abrogated the aberrant activation of the hedgehog pathway. The inhibition of hedgehog signalling prevented bleomycin-induced dermal fibrosis, which is a model for inflammation-dependent fibrosis. In addition, LDE223 was also effective in the tsk-1 model, a model of non-inflammatory stages of fibrosis with autonomous fibroblast activation. ${ }^{17}$ These findings indicate that targeting Smo may be beneficial for different stages of dermal fibrosis. Of particular interest, targeting Smo did prevent the development of experimental fibrosis and induced regression of pre-established fibrosis. In a clinical setting, prevention of fibrosis would be particularly indicated for patients with early progressive disease. In contrast, regression of fibrosis would correspond to reduction of pre-existing damage and potential improvement of organ dysfunction. This would be particularly relevant for patients with pre-existing fibrotic organ damage. Thus, targeting of Smo might theoretically be considered for patients with extensive, long-standing dermal fibrosis. These findings might have direct clinical implications. The role of the hedgehog pathway in multiple human cancers stimulated the development of small-molecule inhibitors against Smo, several of which are currently evaluated in clinical trials (http://www.clinicaltrials.gov). Results from initial studies in humans indicate that pharmacological inhibition of Smo may not be limited by toxicity in adults. ${ }^{67}$ No dose-limiting toxicity occurred in a phase I/II trial with the oral Smo inhibitor GDC449 in doses that efficiently inhibited hedgehog signalling. ${ }^{7}$
Moreover, no grade V events and only one grade VI event occurred in 68 patients and only 1 of 68 patients discontinued GDC449 treatment due to adverse events. However, although no clinical indicators were observed in this trial, inhibition of hedgehog signalling may theoretically interfere with stem cell renewal and may therefore reduce stem cell numbers, for example, in the gut or in the hair follicle. Thus, pharmacological inhibitors of the hedgehog pathway seem to be well tolerated and would be available for clinical trials in scleroderma and in other fibrotic diseases.

In summary, inhibition of hedgehog signalling exerts potent antifibrotic effects in preclinical models of dermal fibrosis and did prevent fibrosis and induced regression of pre-established fibrosis. Since pharmacological inhibitors of Smo are available and seem well tolerated, inhibition of the hedgehog pathway might be a promising approach for novel molecular targeted treatments of dermal fibrosis.

Contributors $A H$ and JHWD designed the study. AH, TK, KP, CD, MT, CC, PZ, CB, AA and $M R$ acquired data. AH, TK, MR, OD, CB, GS and JHWD interpreted the data. AH, $\mathrm{CB}, \mathrm{OD}, \mathrm{GS}$ and JHWD prepared the manuscript.

Acknowledgements The study was supported by the Deutsche Forschungsgesellschaft, grant A20 of the Interdisciplinary Center of Clinical Research (IZKF) in Erlangen, the Career Support Award of Medicine of the Ernst Jung Foundation, CMH Research Project No. 00000023728 and the Immunobone program. LDE223 was kindly provided by Novartis. The authors thank Maria Halter and AnnaMaria Herrmann for excellent technical support.

Competing interests None.

Provenance and peer review Not commissioned; externally peer reviewed.

\section{REFERENCES}

1. Gabrielli A, Avvedimento EV, Krieg T. Scleroderma. N Eng/ J Med 2009;360:1989-2003.

2. Ingham PW, McMahon AP. Hedgehog signaling in animal development: paradigms and principles. Genes Dev 2001;15:3059-87.

3. Ingham PW, Nakano $Y$, Seger $C$. Mechanisms and functions of Hedgehog signalling across the metazoa. Nat Rev Genet 2011;12:393-406.

4. Gallet A. Hedgehog morphogen: from secretion to reception. Trends Cell Biol 2011;21:238-46.

5. Avouac J, Palumbo K, Tomcik M, et al. Inhibition of AP-1 signaling abrogates TGF- $\beta$ mediated activation of fibroblasts and prevents experimental fibrosis. Arthritis Rheum. Published Online First: 2 Dec 2011. doi:10.1002/art.33501

6. Rudin CM, Hann CL, Laterra J, et al. Treatment of medulloblastoma with hedgehog pathway inhibitor GDC-0449. N Engl J Med 2009;361:1173-8.

7. Von Hoff DD, LoRusso PM, Rudin CM, et al. Inhibition of the hedgehog pathway in advanced basal-cell carcinoma. N Engl J Med 2009;361:1164-72.

8. Akhmetshina A, Dees C, Pileckyte M, et al. Dual inhibition of c-abl and PDGF receptor signaling by dasatinib and nilotinib for the treatment of dermal fibrosis. FASEB J 2008;22:2214-22.

9. Palumbo K, Zerr P, Tomcik M, et al. The transcription factor JunD mediates transforming growth factor $\{$ beta $\}$-induced fibroblast activation and fibrosis in systemic sclerosis. Ann Rheum Dis 2011;70:1320-6.

10. Dees C, Akhmetshina A, Zerr P, et al. Platelet-derived serotonin links vascular disease and tissue fibrosis. J Exp Med 2011;208:961-72.

11. Avouac J, Fürnrohr BG, Tomcik M, et al. Inactivation of the transcription factor STAT-4 prevents inflammation-driven fibrosis in animal models of systemic sclerosis. Arthritis Rheum 2011;63:800-9.

12. Minakuchi Y, Takeshita F, Kosaka N, et al. Atelocollagen-mediated synthetic small interfering RNA delivery for effective gene silencing in vitro and in vivo. Nucleic Acids Res 2004;32:e109.

13. Distler JH, Jüngel A, Huber LC, et al. Imatinib mesylate reduces production of extracellular matrix and prevents development of experimental dermal fibrosis. Arthritis Rheum 2007:56:311-22.

14. Reich N, Maurer B, Akhmetshina A, et al. The transcription factor Fra-2 regulates the production of extracellular matrix in systemic sclerosis. Arthritis Rheum 2010;62:280-90.

15. Distler JH, Jüngel A, Caretto D, et al. Monocyte chemoattractant protein 1 released from glycosaminoglycans mediates its profibrotic effects in systemic sclerosis via the release of interleukin-4 from T cells. Arthritis Rheum 2006;54:214-25.

16. Distler JH, Jüngel A, Huber LC, et al. The induction of matrix metalloproteinase and cytokine expression in synovial fibroblasts stimulated with immune cell microparticles. Proc Natl Acad Sci U S A 2005;102:2892-7.

17. Beyer C, Schett G, Distler 0 , et al. Animal models of systemic sclerosis: prospects and limitations. Arthritis Rheum 2010;62:2831-44. 


\section{ARD Inhibition of hedgehog signalling prevents experimental fibrosis and induces regression of established fibrosis}

Angelika Horn, Trayana Kireva, Katrin Palumbo-Zerr, Clara Dees, Michal Tomcik, Cinzia Cordazzo, Pawel Zerr, Alfiya Akhmetshina, Martial Ruat, Oliver Distler, Christian Beyer, Georg Schett and Jörg H W Distler

Ann Rheum Dis 2012 71: 785-789 originally published online March 8, 2012

doi: 10.1136/annrheumdis-2011-200883

Updated information and services can be found at:

http://ard.bmj.com/content/71/5/785

These include:

Supplementary Material

References

Email alerting service
Supplementary material can be found at:

http://ard.bmj.com/content/suppl/2012/03/07/annrheumdis-2011-2008 83.DC1.html

This article cites 17 articles, 6 of which you can access for free at: http://ard.bmj.com/content/71/5/785\#BIBL

Receive free email alerts when new articles cite this article. Sign up in the box at the top right corner of the online article.

Topic
Collections

Articles on similar topics can be found in the following collections

Connective tissue disease (4233)

Immunology (including allergy) (5116)

\section{Notes}

To request permissions go to:

http://group.bmj.com/group/rights-licensing/permissions

To order reprints go to:

http://journals.bmj.com/cgi/reprintform

To subscribe to BMJ go to:

http://group.bmj.com/subscribe/ 